\title{
In erster Linie Fotografie
}

\author{
Hroje Klasić
}

Den Film Walter verteidigt Sarajevo habe ich mir mehrere Male angeschaut. Eine von vielen Szenen, die ich in diesem Film liebe, ist jene, in der Zis (Ljubiša Samardžić) in seinem Fotogeschäft mit den deutschen Soldaten seinen Spott dadurch trieb, dass er beim Fotografieren Worte auf Serbisch sprach, die diese nicht verstehen konnten. Das Filmzitat hat mit dem Buch von Davor Konjikušić zwar nicht viel gemeinsam, doch war für mich interessanterweise die beschriebene Szene die erste Assoziation, als sich Davor bei mir gemeldet hatte, um mir zu erklären, an welchem Buch er arbeitet, und mich darum bat, ein Vorwort zu verfassen. Bereits als zweite Assoziation tauchte vor meinen Augen das Bild des verwundeten Tito am Fluss Sutjeska in Gesellschaft von Ivan Ribar auf (Abb. S. 98, Kap. 4), und anschließend die Aufnahme von Stjepan Filipović in einer Pose, die ohne ein einziges Wort den Begriff Mut am besten zu beschreiben vermag (Abb. S. 162, Kap. 7).

An dieser Stelle enden dann auch meine Assoziationen an die Fotografien aus dem Zweiten Weltkrieg und dem Volksbefreiungskampf. Mir wurde schnell klar, dass meine Kenntnisse über dieses Thema überaus bescheiden waren; um ehrlich zu sein, habe ich die Fotografie als dokumentarisches oder propagandistisches Medium zu keiner Zeit als ein relevantes Thema erlebt. Übrigens wie im alltäglichen Leben. Sie ist immer irgendwie um uns herum präsent, zumeist als Objekt und selten als Subjekt. Die meisten Menschen fassen die Fotografie auch heute als Mittel auf, dessen man sich bedient, um eine Geschichte zu erzählen, und nicht als eine Geschichte für sich. Der Fotograf oder irgendjemand anderes, der hinter dem Objektiv steht, ist zumeist nur Mittler bei der Erzählung der Geschichte und selten auch ihr Autor.

Aufgrund all des oben Genannten, insbesondere jedoch wegen des Enthusiasmus und des Feuers in der Stimme, mit welcher der Autor mir seine Absicht erklärte, beschloss ich, meinen bescheidenen Beitrag zu diesem so bedeutenden Werk zu leisten.

Das Buch, das Davor Konjikušić verfasst hat, ist aus vielerlei Gründen wichtig. Erstens: Positiv über den Volksbefreiungskrieg, die Partisanenbewegung, den Antifaschismus, über Werte zu schreiben, für die Tausende von Mädchen und Jungen gekämpft und ihr Leben gelassen haben, hat heutzutage eine weitaus größere Bedeutung als der Inhalt des Buches, da dies in der modernen kroatischen Gesellschaft nicht mehr allein Forschungsleistung und publizistischer Akt ist. Es ist zu einem weiten Teil auch ein Akt des Aktivismus, der bürgerlichen Verantwortung, leider auch der Zivilcourage. 
Zweitens: Das Buch ist auch wegen des Themas wichtig, es zeigt, wo unsere Forschung zum Zweiten Weltkrieg hätte stehen können und stehen müssen, aber auch wie es damit leider in Wirklichkeit bestellt ist. Über den Zweiten Weltkrieg wurden Tausende von Büchern in zahlreichen Sprachen verfasst, doch ihr vorrangiges Interesse galt der militärischen und der politischen Geschichte. Es gibt keine Zweifel daran, dass zahlreiche Standpunkte und Interpretationen - insbesondere in den Arbeiten einheimischer Autoren - einer wissenschaftlich fundierten Revision unterzogen werden müssten. Des Weiteren gibt es auch eine ganze Reihe von Geschehnissen, die im sozialistischen Jugoslawien niemals bearbeitet wurden, so zum Beispiel Akte der Vergeltung und Verbrechen, die seitens der Sieger gegen Ende und unmittelbar nach Kriegsende begangen worden sind. Unter Berücksichtigung aller "Unterlassungen" und ideologisierter Diskurse sind die meisten Fakten über den Zweiten Weltkrieg auf jugoslawischem Gebiet zumindest vom politischen und militärischen Aspekt her wohl bekannt. Das, was fehlt und was eine logische Fortsetzung des Forschungsinteresses hätte darstellen sollen, ist die Untersuchung der gesellschaftlichen und wirtschaftlichen Geschichte, des Alltags, der Gender History und Ähnlichem.

Während Forscher weltweit bereits vor langer Zeit diesen qualitativen Schritt nach vorn gingen, wurde auf dem Gebiet des ehemaligen Jugoslawien ebenfalls ein großer qualitativer Schritt gemacht, jedoch nach hinten. Mit den erwähnten Themen hat sich nicht nur kaum jemand beschäftigt, es überwiegen inzwischen auch Arbeiten, die ein typisches Beispiel für politisch motivierten Revisionismus darstellen. Und so werden Bücher veröffentlicht, die den Nachweis über den antifaschistischen Charakter der Tschetnik-Bewegung und den verbrecherischen Charakter der Partisanenbewegung zu erbringen versuchen, Bücher, die die politischen und militärischen Anführer der Vasallenstaaten glorifizieren und im Gegenzug die militärischen und politischen Anführer der Volksbefreiungsbewegung dämonisieren. Der Holocaust und Genozid werden relativiert, der Charakter des Konzentrationslagers Jasenovac ebenso negiert wie der Befreiungs- und Modernisierungscharakter des Partisanenkampfes.

Die Art und Weise der Auseinandersetzung mit der Vergangenheit ist ursächlich mit der allgemeinen gesellschaftlichen Situation verbunden. Die politischen Eliten machen sich bestimmte Interpretationen der Vergangenheit manchmal zunutze, doch nicht selten werden sie von ihnen auch initiiert. Die Folge dessen sind mehrere Tausend niedergerissener Partisanendenkmäler, tausende vernichteter Bücher über den Volksbefreiungskampf, die Umbenennung von Straßen, die früher die Namen der Helden des Partisanenkrieges trugen, aber auch - was am wichtigsten ist - die Schaffung eines Klimas der Scham anstatt des Stolzes. Gleichzeitig trug eine revisionistisch geprägte Herangehensweise an die Ustascha-Bewegung und den Unabhängigen Staat Kroatien zu einer relativierenden Haltung gegenüber dieser düstersten Epoche der kroatischen Geschichte bei. Über Nacht war es in Kroatien absolut normal geworden, dass auf Straßen und in Sportstadien Parolen und Grüße der Ustascha erschallten, dass militärische Einheiten im Vaterlandskrieg nach Kommandanten der Ustascha sowie Straßen nach Politikern der Ustascha-Bewegung benannt wurden. Der Staat selbst sponserte Feierlichkeiten aus Anlass der Jahrestage der Ustascha-Armee und nicht der Partisanen. 
Aus diesem Grund möchte ich auch zu meinem ursprünglichen Gedanken zurückkehren. In der heute vorherrschenden Atmosphäre besitzt jedes Buch, jeder noch so geringe Beitrag, der sich dem Revisionismus widersetzt und der die Öffentlichkeit stichhaltig an die Werte des Volksbefreiungskampfes erinnert, einen hohen Stellenwert. Wenn es zudem den eingangs erwähnten qualitativen Schritt nach vorn mit sich bringt und sich der Schwerpunkt der Forschung von der militärischen und politischen Geschichte auf einen weniger bekannten Bereich verlagert, handelt es sich um einen außerordentlich wertvollen historiografischen und gesellschaftlichen Beitrag.

Doch es gibt auch einen dritten Grund. Dieses Buch verdeutlicht das Bedürfnis, ja sogar die Notwendigkeit eines interdisziplinären Ansatzes bei der Untersuchung eines bestimmten Themas. Und obwohl dies die logische Vorgehensweise eines jeden Forschers sein müsste, ist das leider häufig nicht der Fall. Davor Konjikušić hat seinen Hochschulabschluss an der Akademie für Dramaturgie erlangt. Sein primäres Interesse gilt der Fotografie als Medium, mit dem er die unterschiedlichsten soziologisch-politischen Beziehungen untersucht. Er ist gleichzeitig jedoch auch ein etablierter Journalist, der in seinen Artikeln die verschiedenen Herausforderungen diskutiert, mit denen sich die moderne Gesellschaft heute konfrontiert sieht. In beiden Fällen beschäftigen ihn vorrangig jene Themen, die just in dem Augenblick der Auseinandersetzung hochaktuell sind. Trotz allem beschloss er, ein Buch über mehr als siebzig Jahre zurückliegende Geschehnisse zu schreiben. Zudem wählte er, wie ich bereits zu betonen versuchte, eines der komplexesten, kontroversesten und einflussreichsten Ereignisse des 20. Jahrhunderts, das mit seinem Inhalt und seinen Folgen trotz oder gerade wegen der verstrichenen Zeit auch das Leben der Menschen im 21. Jahrhundert nicht aufhört zu beeinflussen.

Und er hat eine vorzügliche Arbeit geleistet.

Im Vordergrund des Buches steht die Fotografie als Dokument der Zeit und als historische Quelle, aber auch als Mittel und Ziel des künstlerischen Ausdrucks. Indem er in die verborgensten Ecken der Museumsdepots und privaten Familiensammlungen vorstieß, begann der Autor, ähnlich einem erfahrenen Archäologen, mit Hilfe der Fotografien große Schlachten ebenso wie kleine menschliche Schicksale zu rekonstruieren. Gegenstand seines Interesses waren angesehene Politiker und militärische Befehlshaber, aber auch gemeine Soldaten. Den sogenannten ikonischen Fotografien, das heißt jenen Fotografien, die zu Symbolen einer Zeit geworden sind, schenkte er die gleiche Aufmerksamkeit wie jenen, die »im Vorbeigehen«, ja fast zufällig entstanden sind. Besonders interessant und innovativ macht das Buch die Hommage auf die Schöpfer der Fotografien. Unter ihnen gab es Profis, wahre Künstler, aber auch Amateure, die ihre fotografischen Fertigkeiten gerade in jener der Kunst und der Kultur keineswegs zugeneigten Zeit weiterentwickelt haben. Über die Fotografen, ihre Schicksale und späteren Karrieren weiß man heute für gewöhnlich kaum etwas. Der Autor unternahm den Versuch, diese Ungerechtigkeit geradezurücken, denn die Mehrheit der Fotografien übersteigt mit ihrer Bedeutung und ihrem Wert die Relevanz ihrer Schöpfer, doch gleichzeitig gäbe es keine einzige Fotografie ohne denjenigen, der sie aufzunehmen beschloss. Ohne seine oder ihre Auswahl des Themas, der Ausschnitte, der Perspektive oder einfach ohne jenes »Klicken«, mit dem ein vergängliches Ereignis verewigt wurde. 
Ein zweites Thema ist der Krieg, der im Hinblick auf die Zahl der Opfer und der Akteure der größte und der schrecklichste in der Geschichte der Menschheit war. Das Thema ist genauer gesagt der Zweite Weltkrieg auf dem Gebiet Jugoslawiens, oder noch präziser, der Volksbefreiungskampf der Partisanen auf der einen Seite sowie die Okkupation und Kollaboration auf der anderen Seite. Obwohl das Buch interessante Details aus der politischen und militärischen Geschichte enthält, ist seine Grundmotivation weitaus »bodenständiger«. Konjikušić versucht, mit Hilfe der aufgenommenen Fotografien dem Leser die Atmosphäre vor Augen zu führen, die Leiden und die Aufopferung der Menschen, den Terror und die Solidarität, den Pragmatismus und Idealismus sowie den Mut und den Verrat darzustellen. Der Wandel der Haltung gegenüber der Fotografie wurde dazu genutzt, die Entwicklung der Revolution aufzuzeigen. Die anfänglichen Irrungen, Entbehrungen und die fehlende klare Strategie sollten später in einem gut geleiteten und wohl durchdachten Projekt münden. Es scheint, als wollte der Autor den Mangel an Kameras, Chemikalien und Fotopapier mit dem Mangel an Waffen und Munition in Verbindung bringen. Auf der anderen Seite sind für ihn die zeitlosen Fotografien, die die Geschichte von David und Goliath auch über die Grenzen des Kriegsschauplatzes hinausgetragen haben, ein unverzichtbares Steinchen im Mosaik des gesamten (militärischen) Sieges.

Konjikušić verdeutlicht eine überaus interessante Haltung der Partisanen zur Fotografie. Das anfängliche Misstrauen, ja sogar die Geringschätzung der Fotografie entwickelte sich mit der Zeit zu einer hochprofessionellen Beziehung. Bis zum Ende des Krieges wurden Aufnahmen mit unterschiedlichsten Inhalten zu ideologisch-propagandistischen und außenpolitischen Zwecken genutzt. Der Sieg wäre auch ohne Fotomaterial errungen worden, doch mit ihm wurde er sichtbar, noch lange bevor es wirklich zum Sieg gekommen war.

Der dritte Bestandteil des Buches ist der Autor des Buches selbst - Davor Konjikušić, Künstler und Kunstkritiker, Forscher und Chronist, Antifaschist und Humanist. Durch die Wahl des Themas und die Art und Weise seiner Auseinandersetzung ist er all den angeführten »Titeln« gerecht geworden, er hat aber auch angedeutet, Talent und Eros für vieles andere zu besitzen. Seine Handschrift ist klar, während seine Blickwinkel eindeutig und seine Schlüsse minutiös sind. Er verteidigt eine Idee, eine Weltanschauung, ohne ihr jedoch zu schmeicheln. Er weiß Errungenschaften zu schätzen, kritisiert aber gleichzeitig jegliche Ausschweifungen. Indem er über die Geschichte schreibt, spricht der Autor auch über die Gegenwart und warnt vor der Zukunft. Obwohl hinter seinen Forschungen eine langwierige, ernsthafte und anspruchsvolle Arbeit steckt, ist deutlich spürbar, dass er das gesamte Unterfangen genossen hat. Einem wahren intellektuellen Homo ludens ähnlich, betrat er fremde Dachböden, pustete den Staub von vergilbten Fotoalben weg, suchte ungeduldig nach neuen, bis dato unbekannten Fotografien und Geschichten, die sich hinter ihnen verbargen. Trotz der schwierigen Thematik sind seine Observationen von Positivismus und Anekdoten aus dem Leben zumeist unbekannter Menschen geprägt, die ihrerseits Geschichte geschrieben haben. Ungeachtet seines Wissens, das durch Tatsachen und überprüfte Informationen untermauert ist, versucht der Autor - von einigen Ausnahmen abgesehen - in der Art eines wahren, von aufrichtigem Idealismus inspirierten Aktivisten den Leser niemals zu überreden oder von etwas zu überzeugen. Vielmehr möchte er ihm einen Weg aufzeigen, von dem er glaubt, dass er der richtige sei. 
Mit diesem Buch hat Davor Konjikušić keineswegs die Tür für künftige Forschungen verschlossen, weder für seine eigenen, noch für die anderer Autoren. Ganz im Gegenteil stellt das Buch eine Einladung und Motivation sowie einen Wegweiser für alle potenziellen Forscher dar. Das Buch ist als großer geschichts- und kunstgeschichtlicher Beitrag zu betrachten, doch ist es nicht nur für Fachleute bestimmt. Ich bin mir absolut sicher, dass es mit seinem Inhalt und Stil das Interesse der breitesten Öffentlichkeit wecken wird.

Das Buch liefert zahlreiche Antworten, wirft aber auch einige gänzlich neue Fragen auf. Das Buch kann genossen werden, indem man es liest oder sich die Fotografien anschaut. Doch sein Hauptanliegen besteht darin, den Leser zum Nachdenken zu bewegen und unsere Auseinandersetzung mit der ungewissen Zukunft im Rückblick auf die rühmliche Vergangenheit zu befördern. 
